\title{
The Puzzling Negative Orbit-Period Derivative of the Low-Mass X-Ray Binary 4U 1820-30 in NGC 6624
}

\author{
M. Peuten ${ }^{1}$, M. Brockamp ${ }^{1,2}$, A.H.W. Küpper ${ }^{3,4}$, P. Kroupa ${ }^{5}$ \\ mpeuten@mpeuten.de (MP), brockamp@astro.uni-bonn.de (MB), akuepper@astro.columbia.edu \\ (AHWK), pavel@astro.uni-bonn.de (PK)
}

\begin{abstract}
$4 \mathrm{U} 1820-30$ is a low-mass X-ray binary near the center of the globular cluster NGC 6624 consisting of, at least, one neutron star and one helium white dwarf. Analyzing 16 years of data from the Rossi X-ray Timing Explorer (RXTE) allows us to measure its orbital period and its time derivative with unprecedented accuracy to be $P=685.01197 \pm 0.00003$ s and $\dot{P} / P=$ $-5.3 \pm 0.3 \times 10^{-8} \mathrm{yr}^{-1}$. Hence, we confirm that the period derivative is significantly negative at the $>17 \sigma$ level, contrary to theoretical expectations for an isolated X-ray binary. We discuss possible scenarios that could explain this discrepancy, and conclude that the center of NGC 6624 most likely contains large amounts of non-luminous matter such as dark remnants. We also discuss the possibility of an IMBH inside NGC 6624, or that a dark remnant close to $4 \mathrm{U}$ 1820-30 causes the observed shift.
\end{abstract}

Subject headings: X-rays: binaries individual (4U 1820-30) — globular clusters: individual (NGC 6624)

\section{Introduction}

The low-mass X-ray binary (LMXB) 4U 182030 is the most prominent $\mathrm{X}$-ray source in the globular cluster NGC 6624. The LMXB shows a complex variability, it has a 171 day periodicity with a luminosity variation of about a factor of three (Priedhorsky \& Terrell 1984). Moreover, it is the first source where X-ray Type I bursts have been observed (Grindlay et al. 1976). These X-ray bursts are only seen when $4 \mathrm{U} 1820-30$ is in low state. Besides the Type I bursts also one superburst was detected so far Strohmaver \& Brown

\footnotetext{
${ }^{1}$ Argelander-Institut für Astronomie, Universität Bonn, Auf dem Hügel 71, 53121 Bonn, Germany

${ }^{2}$ Member of the International Max Planck Research School (IMPRS) for Astronomy and Astrophysics at the University of Bonn and Cologne

${ }^{3}$ Department of Astronomy, Columbia University, 550 West 120th Street, New York, NY 10027, USA

${ }^{4}$ Hubble Fellow

${ }^{5}$ Helmholtz-Institut für Strahlen- und Kernphysik (HISKP), University of Bonn, Nussallee 14-16, 53115 Bonn, Germany
}

2002). Another feature of this atoll source is that it exhibits low frequency (Stella et al. 1987b) and kilohertz (Smale et al. 1997) quasi-periodic oscillations (QPOs).

The feature of $4 \mathrm{U}$ 1820-30 which is relevant for this paper is the $685 \mathrm{~s}$ (11 min) variability (Stella et al. 1987a), with a peak-to-peak modulation amplitude of about $(1.95 \pm 0.03) \%$ in the $\mathrm{X}$-ray regime. This period is generally believed to be caused by the orbital motion of the secondary white dwarf around the more massive neutron star (Rappaport et al. 1987). It is also observed in the ultraviolet (Anderson et al. 1997) with a peak-to-peak amplitude of $16 \%$. Further measurements (Sansom et al. 1989; Tan et al. 1991; van der Klis et al. 1993b a) of the $685 \mathrm{~s}$ periodicity showed that its period derivative seems to be negative. The last measured value by Chou \& Grindlay (2001) $\dot{P} / P=(-3.47 \pm 1.48) \times$ $10^{-8} \mathrm{yr}^{-1}$ is negative, but due to the large uncertainty a positive value cannot be excluded at high confidence.

NGC 6624 is a bulge globular cluster, approxi- 
mately $1.2 \mathrm{kpc}$ (Harris 1996) away from the Galactic Center and $7.9 \mathrm{kpc}$ (Harris 1996) away from Earth. According to the Harris catalog, NGC 6624 has a heliocentric radial velocity of $\left\langle\nu_{r}\right\rangle=53.9 \pm$ $0.6 \mathrm{~km} \mathrm{~s}^{-1}$ and a radial velocity dispersion of $\sigma_{\nu} \approx$ $8.6 \mathrm{~km} \mathrm{~s}^{-1}$. Like most of its bugle/disk counterparts, the cluster is metal-rich with an iron abundance of $[\mathrm{Fe} / \mathrm{H}]=-0.69 \pm 0.02$ (Valenti et al. 2011). But NGC 6624 is very compact, its halflight radius is $r_{h}=49 " 2 \approx 1.88 \mathrm{pc}$ (Harris 1996), and it appears to be core-collapsed with a radial density profile that has a power-law cusp with a slope of about -0.8 to -0.3 Sosin \& King 1995; Novola \& Gebhardt 2006). Early measurements gave a mass density of the core of about $\rho_{c} \approx 1.1 \times$ $10^{5} \mathrm{M}_{\odot} \mathrm{pc}^{-3}$ (Canizares et al. 1978) and a luminosity density of about $\log _{10}\left(\rho_{0}\right)=5.25 \mathrm{~L}_{\odot} \mathrm{pc}^{-3}$ (Piotto et al. 2002).

One of the first theoretical descriptions of $4 \mathrm{U}$ 1820-30 was done by Rappaport et al. (1987) who described the LMXB as consisting of a neutron star and a degenerate almost pure, or entirely pure, helium dwarf. According to this interpretation, the secondary fills its Roche lobe and thus transfers matter to the accretion disc of the neutron star. This theory was later extended by Arons \& King (1993) who showed that the high ultraviolet luminosity could be explained by a reprocessing of the emitted X-ray photons. One of the observations which had not been explained by Rappaport et al. (1987), the 171 days period, was later explained by Prodan \& Murray (2012), who took up the idea of a third bound star. This scenario had already been suggested by Grindlay (1988) and again by Chou \& Grindlay (2001). Prodan \& Murray (2012) calculated the properties needed for the additional star to modulate the accretion rate via the Kozai mechanism.

To date, many of the predictions made by Rappaport et al. (1987) have been confirmed by observations, but there is one important prediction that is not met yet: Rappaport et al. (1987) predict that the period derivative of the $685 \mathrm{~s}$ period must be positive, as the secondary is losing mass to the neutron star. The X-rays which are detected from $4 \mathrm{U}$ 1820-30 are a direct consequence of this process. That is, the orbital period of the secondary should be increasing with time. Rappaport et al. (1987) further predict a value of around $\dot{P} / P \approx 8.8 \times 10^{-8} \mathrm{yr}^{-1}$ for the period derivative. When calculating this value, the authors neglected some effects (see Prodan \& Murray (2012) for a discussion) which could lower the intrinsic period derivative, but these effects should not change the sign of $\dot{P} / P$. Thus, the period derivative should nevertheless be positive, but the observed value seems to be negative. However, as already stated, the latest measurement does not exclude at a high significance that the true value could be positive.

The motivation of the present paper is to confirm the existence of a negative period derivative with higher statistical significance than before. To achieve this goal we analyzed sixteen years of Rossi X-ray Timing Explorer (RXTE) data, which had not been analyzed in this respect so far. We are now able to show with an updated value of $\dot{P} / P=(-5.3 \pm 0.3) \times 10^{-8} \mathrm{yr}^{-1}$ that, contrary to the theoretical predictions, the period derivative is truly negative at high confidence. It is smaller than the result from Chou \& Grindlav (2001) but equal to the value that van der Klis et al. (1993a) had measured before.

In Section 2, we give an overview about the observations. In the same section, we also present a detailed description of the different steps involved in the reduction of the $\mathrm{RXTE} / \mathrm{PCA}$ data. The subsequent Section 3 describes our analysis of the results from the reduction, graphically presented in Fig. 4, and our calculation of the period derivative as being $\dot{P} / P=(-5.3 \pm 0.3) \times 10^{-8} \mathrm{yr}^{-1}$. In Section 4. we discuss some possible explanations for the difference between theoretical predictions and the value we measured: We conclude that this difference is primarily generated by gravitational acceleration within the cluster. We therefore discuss in this section the probable sources of such a gravitational acceleration such as an Intermediate Mass Black Hole (IMBH), a high central concentration of dark remnants or a close flyby of a single dark remnant such as a stellar-mass black hole or neutron star. A summary of our conclusions can be found in Section 5 .

\section{Data Reduction and Analysis}

\subsection{Available Datasets}

The Rossi X-ray Timing Explorer (RXTE) satellite was launched in December 1995 and the mission lasted for 16 years until January 2012. 
The primary aim of RXTE was to analyze the Xray universe with a high temporal resolution and a medium energy resolution. The RXTE consists of three instruments. We here use data from the Proportional Counter Array (PCA) (Jahoda et al. 1996, 2006). The PCA has a high temporal resolution of $1 \mu$ s over an energy range of $2-60 \mathrm{keV}$ with an energy resolution of $18 \%$ (at $6 \mathrm{keV}$ ).

Parts of the 1996 and 1997 data were already analyzed by Chou \& Grindlav (2001), but for the sake of comparability and because of the fact that, in the meantime, newer reduction software and calibration data became available (Shaposhnikov et al. 2012), we reevaluated them in this paper. We adopted the analysis approach from Chou \& Grindlav (2001) as they worked with the same satellite and the same target.

For our analysis we used all available observations of $4 \mathrm{U} 1820-30$ made by the PCA. These 261 observations were made throughout the whole lifetime of the RXTE mission, which includes data from 1996 to 2011. Fig. [1] shows a plot of the measurements of 4U 1820-30 from the All-Sky Monitor (ASM, Levine et al. 1996), which was also on board the RXTE mission, together with bars indicating the time when observations were made with the PCA instrument. It is apparent that almost half of all observations were made in the first four years of the mission. In the remaining twelve years, the PCA instrument observed 4U 1820-30 mainly when it was in low state, in order to catch some of the X-ray Type I bursts. As previous publications (for example Morgan et al. 1988) showed no correlation between the relative amplitude of the $685 \mathrm{sec}$ periodicity and the overall X-ray luminosity, the results from these years are subject to a lowered statistic.

From the available PCA modes we analyzed the STANDARD-2 data as it is available from all observations. The PCA STANDARD-2 data has a time resolution of $16 \mathrm{~s}$ and an energy resolution of 129 bit covering the whole PCA energy range from 2 to $60 \mathrm{keV}$. The PCA instrument consists of five equal Proportional Counter Units (PCU). Throughout the mission these five units performed quite differently. For this project we decided to only use data from PCU2 which was not only the most stable one but also in many observations the only observing one. There were only two observations (Observations 40017-01-71-00 and 40017-
01-17-01) where PCU2 was deactivated due to an earlier breakdown. We therefore decided to use data from PCU0 for these time ranges. Until May 2000, when PCU0 was hit by a micrometeorite, it performed as well as PCU2.

To exclude energy specific systematics, we performed the complete analysis in different energy ranges $(1-5 \mathrm{keV}, 5-8 \mathrm{keV}, 8-20 \mathrm{keV}, 20-60 \mathrm{keV}$ and full PCA energy range), but found, as expected from previous observations, no correlation between the results and the selected energy range. For this reason we present here the analysis of the complete energy band $(2-60 \mathrm{keV})$ of the PCA instrument. In the reduction and the following analysis we used the HEASOFT V6.12 (as of March 12, 2012) 1 software.

\subsection{Data Reduction}

We started the reduction by filtering the raw observational data for "bad times". Bad times are periods with some sort of interference, like Earth occultation, passage of the South Atlantic Anomaly or any other events which can interfere with the detector. Next, the resulting light curves were inspected for anomalies such as X-ray bursts. Since these bursts interfere with the $685 \mathrm{sec}$ signal, they had to be removed from the data. For the one observation (Observation 30057-01-04-08G) where the superburst was observed, we decided to remove the whole observation because the superburst almost dominates the entire observation. All in all we found eighteen X-ray bursts in the RXTE data, including the superburst. After identifying all unusable time periods, we reran the filtering step, but this time by leaving out inapplicable data. In the next step, we generated the background for the different observations (see Jahoda et al. 2006 for $\mathrm{a}$ in depth description) and then we subtracted it from the associated observations. Afterwards, the data was corrected for barycentric arrival times $\left(\mathrm{BJD}_{\mathrm{BDT}}\right)$. This is required to have a locationindependent time stamp on all data.

\subsection{Data Analysis}

The next step of the analysis consists of epoch folding the individual light curves. But when comparing the length of the different observations, we

1 https://heasarc.gsfc.nasa.gov/lheasoft/ 

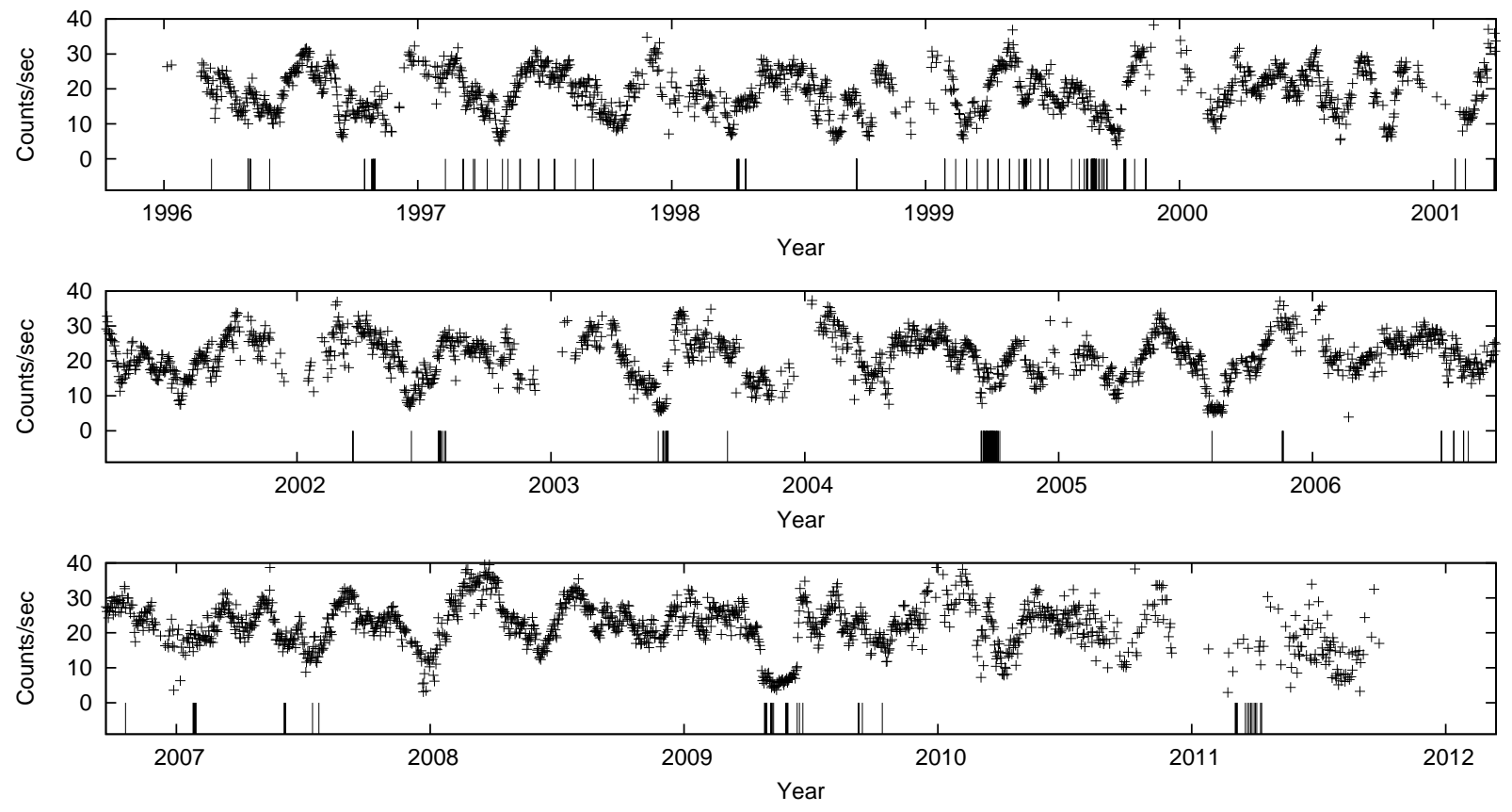

Fig. 1.- Plot showing the distribution of the observations over the whole lifetime of the RXTE mission. The black crosses are the ASM counts of 4U 1820-30 while each black bar represents a PCA observation of 4U 1820-30.

found that there are many short observations, i.e. shorter than one RXTE orbit $\approx 90 \mathrm{~min}$. There were even ten light curves which not even covered the whole $685 \mathrm{sec}$ period. However, many of these short observations were taken on the same day. We therefore decided to join together the light curves of observations being no further away from each other than 24h. This improves the results from the epoch folding method as this technique needs more than one complete period of $685 \mathrm{sec}$ to give an improved result. The averaged observation length of all resulting 154 light curves was $2.6 \mathrm{~h}$, of which none were shorter than the $685 \mathrm{sec}$ period of the LMXB. Before folding the light curves they got rebinned. Following Chou \& Grindlav (2001), we chose 32 phase bins, as varying this value did not give any improvements. Afterwards, these light curves were finally folded with the ephemeris by Tan et al. (1991):

$$
\begin{gathered}
T_{N}^{\max }=\mathrm{HJD}_{\mathrm{UTC}} 2442803.63544 \\
+\left(\frac{685.0118 \mathrm{~s}}{86400 \mathrm{~s}}\right) \times N
\end{gathered}
$$

where $T_{N}^{\max }$ is the time for the maximum of the $N$-th cycle. Since our data had BJD BDT time stamps, we had to convert the above formula (see Eastman et al. 2010) to:

$$
\begin{gathered}
T_{N}^{\max }=\quad \mathrm{BJD}_{\mathrm{TDB}} 2442803.63600 \\
+\left(\frac{685.0118 \mathrm{~s}}{86400 \mathrm{~s}}\right) \times N
\end{gathered}
$$

In Fig. 2 we present one of the resulting folded light curves. The sine-like behavior of the $685 \mathrm{sec}$ period, as also found by all previous analysis, is clearly evident. In some of the folded light curves we also detected a second minimum as shown in Fig. 3. This second minimum was first observed by Morgan et al. (1988) and later by Sansom et al. (1989), Tan et al. (1991) and van der Klis et al. (1993b,a). This minimum seems to appear randomly with changing positions and amplitude. Moreover, as the observation times are randomly distributed, we performed no extensive analysis of this phenomenon.

In order to determine the phase of the maximum we approximated a sine-function to the folded light curves. The results are also shown in Fig. 2 and 3. For one folded light curve (from Observation 90027-01-01-03, September 13, 2004), a sine curve approximation failed and this data 


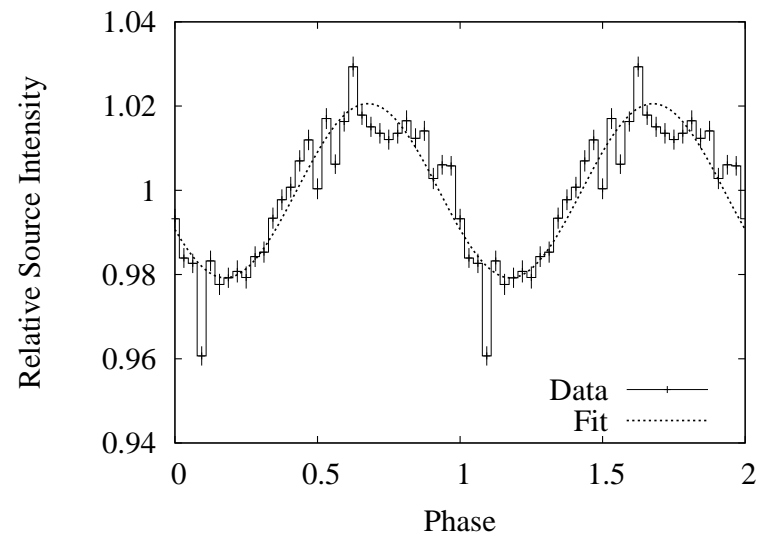

Fig. 2.- Folded light curve from Observation 40017-01-05-00: The solid black histogram is the folded light curve with $1 \sigma$ errors. The dotted black curve is the best fit sine function. One can clearly see the sine-like behavior. The obtained phase value is: $0.68 \pm 0.01$.

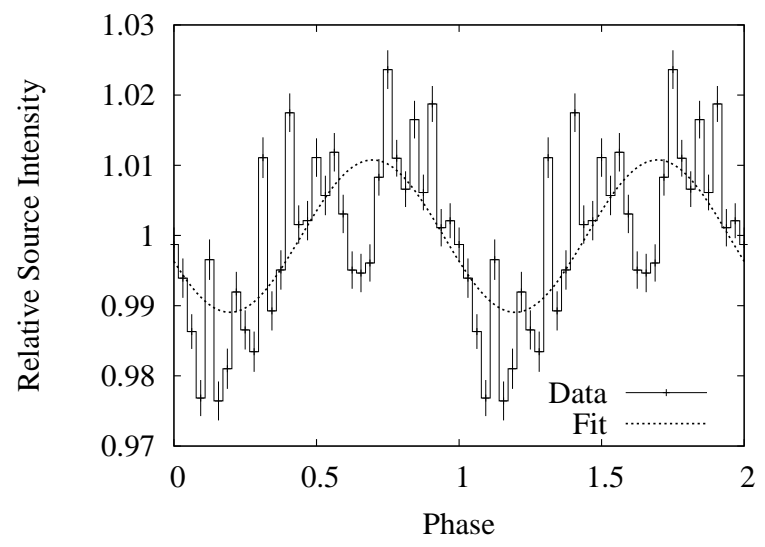

Fig. 3. - Folded light curve from Observation 40017-01-04-00: The solid black histogram is the folded light curve with $1 \sigma$ errors. The dotted black curve is the best fit sine function. Besides the sinelike behavior, one can clearly see the second dip. The obtained phase value is: $0.70 \pm 0.04$ point was therefore removed. As the phase is periodic we took care that the resulting values were as continuous to each other and to previous results as possible. This is a reasonable assumption, as there were no indications against it. The sine fit allows us to also determine the average modulation amplitude (peak to peak). We measured $(1.95 \pm 0.03) \%$.

Following Chou \& Grindlay (2001) the obtained phases from the same year were joined together. This was done by weighted averaging the individual phase values from the same year. To deal with the scattering of individual phases around the mean value, we quadratically added the mean phase jitter, which is the weighted standard deviation of the phase values, to the mean phase error. The results from this last step are given in Table 1 and are presented in Fig. 4. Besides our results, we also listed and plotted the results from previous publications as listed by van der Klis et al. $(1993 \mathrm{a}, \mathrm{b})$. These datasets come from the following X-ray missions: SAS-3 (Morgan et al. 1988), Ariel V (Smale et al. 1987), Einstein (Morgan et al. 1988), Tenma (Sansom et al. 1989), EXOSAT (Stella et al. 1986), GINGA (Sansom et al. 1989, Tan et al. 1991 and van der Klis et al. 1993b) and ROSAT (van der Klis et al. 1993b. a). In order to combine our results with those datasets, we first had to convert their time stamps into BJD $\mathrm{TDB}_{\text {. }}$ With the reduced and analyzed RXTE data at hand, together with the data from previous missions adapted to our requirements, we can now determine the proper value of $\dot{P} / P$. This will be the topic of the next Section 3 .

\section{Results}

In Fig. 4 one sees how the phase shift evolves over time. The data were folded with the linear ephemeris as given by Tan et al. (1991) (see also Eq. 2). If the true ephemeris would be really linear, one would expect the phase shift to be constant. This is apparently not the case: the phase shift is decreasing. This behavior can be approximated very well with the true ephemeris having a quadratic time dependence. The observed phase shift in Fig. 4 is therefore the difference between the two ephemerides. Following Chou \& Grindlay (2001), we subtracted Eq. 2 from a quadratic 


\begin{tabular}{|c|c|c|c|}
\hline Arrival time of Maximum [BJD $\left.{ }_{\mathrm{TDB}}\right]$ & Phase & Satellite & Reference \\
\hline 2442803.64 & $0.01 \pm 0.03$ & SAS-3 & 1 \\
\hline 2442866.86 & $-0.01 \pm 0.04$ & SAS-3 & 1 \\
\hline 2442876.81 & $0.02 \pm 0.04$ & SAS-3 & 1 \\
\hline 2442889.19 & $0.04 \pm 0.09$ & Ariel V & 2 \\
\hline 2443018.64 & $0.03 \pm 0.05$ & SAS-3 & 1 \\
\hline 2443050.95 & $0.00 \pm 0.02$ & SAS-3 & 1 \\
\hline 2443196.93 & $0.04 \pm 0.05$ & SAS-3 & 1 \\
\hline 2443406.11 & $0.07 \pm 0.03$ & SAS-3 & 1 \\
\hline 2443969.20 & $0.05 \pm 0.06$ & Einstein & 1 \\
\hline 2445554.11 & $0.06 \pm 0.06$ & Tenma & 3 \\
\hline 2445969.88 & $0.01 \pm 0.03$ & EXOSAT & 4 \\
\hline 2446172.22 & $0.03 \pm 0.03$ & EXOSAT & 4 \\
\hline 2446297.41 & $0.13 \pm 0.07$ & EXOSAT & 4 \\
\hline 2446331.57 & $0.05 \pm 0.03$ & EXOSAT & 4 \\
\hline 2446916.51 & $-0.02 \pm 0.03$ & GINGA & 3 \\
\hline 2447569.84 & $-0.08 \pm 0.03$ & GINGA & 5 \\
\hline 2447641.36 & $-0.13 \pm 0.03$ & GINGA & 5 \\
\hline 2448327.96 & $-0.13 \pm 0.03$ & GINGA & 6 \\
\hline 2448328.42 & $-0.08 \pm 0.040$ & ROSAT & 6 \\
\hline 2449057.60 & $-0.12 \pm 0.06$ & ROSAT & 7 \\
\hline 2449071.40 & $-0.12 \pm 0.02$ & ROSAT & 7 \\
\hline 2450299.23 & $-0.23 \pm 0.06$ & RXTE & This work \\
\hline 2450584.90 & $-0.27 \pm 0.07$ & RXTE & $"$ \\
\hline 2450960.23 & $-0.31 \pm 0.03$ & RXTE & $"$ \\
\hline 2451378.59 & $-0.34 \pm 0.07$ & RXTE & $"$ \\
\hline 2451978.78 & $-0.47 \pm 0.06$ & RXTE & $"$ \\
\hline 2452453.69 & $-0.6 \pm 0.1$ & RXTE & $"$ \\
\hline 2452815.24 & $-0.6 \pm 0.1$ & RXTE & $"$ \\
\hline 2453273.76 & $-0.65 \pm 0.08$ & RXTE & $"$ \\
\hline 2453659.48 & $-0.65 \pm 0.05$ & RXTE & $"$ \\
\hline 2453961.10 & $-0.80 \pm 0.04$ & RXTE & $"$ \\
\hline 2454201.79 & $-0.89 \pm 0.07$ & RXTE & $"$ \\
\hline 2454991.88 & $-1.0 \pm 0.2$ & RXTE & $"$ \\
\hline 2455643.48 & $-1.08 \pm 0.07$ & RXTE & $"$ \\
\hline
\end{tabular}

Table 1: Results from previous missions as listed by van der Klis et al. (1993a, $\mathrm{b}$ ) converted to BJD $\mathrm{TDB}_{\text {vime }}$ stamps together with results from our analysis of the RXTE data from 4U 1820-30. The error values for the arrival times are below $10^{-3}$ days and are therefore not listed. Note: The resulting RXTE phase values are chosen to be continuous with values from the previous publications. References: (1) Morgan et al. (1988); (2) Smale et al. (1987); (3) Sansom et al. (1989); (4) Stella et al. (1987a); (5) Tan et al. (1991); (6) van der Klis et al. (1993b); (7) van der Klis et al. (1993a). 


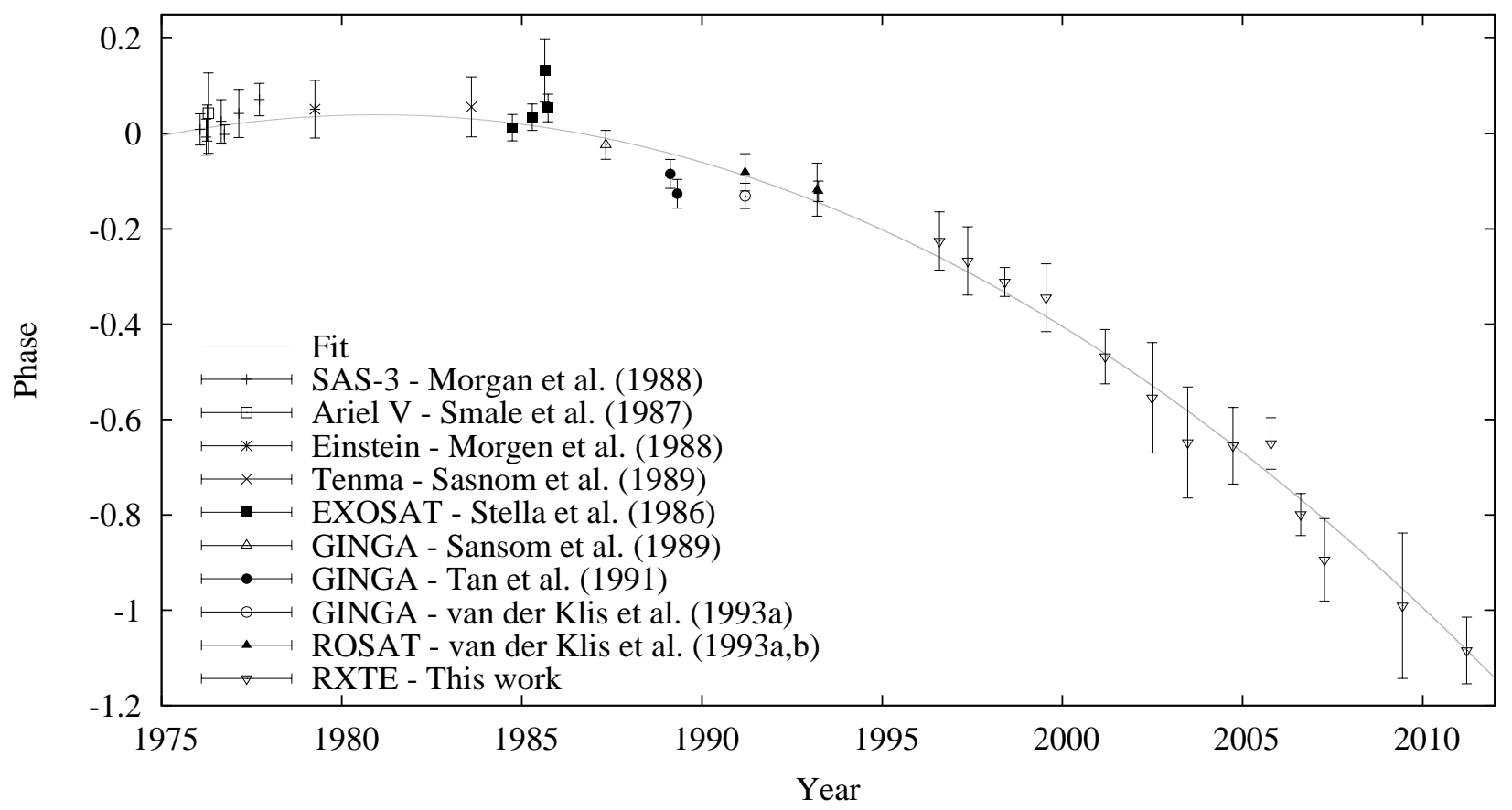

Fig. 4.- Phases of the $685 \mathrm{sec}$ modulation of 4U 1820-30 from X-ray satellite observations from 1976 to 2011. The solid line represents the best quadratic fit from which the new value for the period derivative is determined.

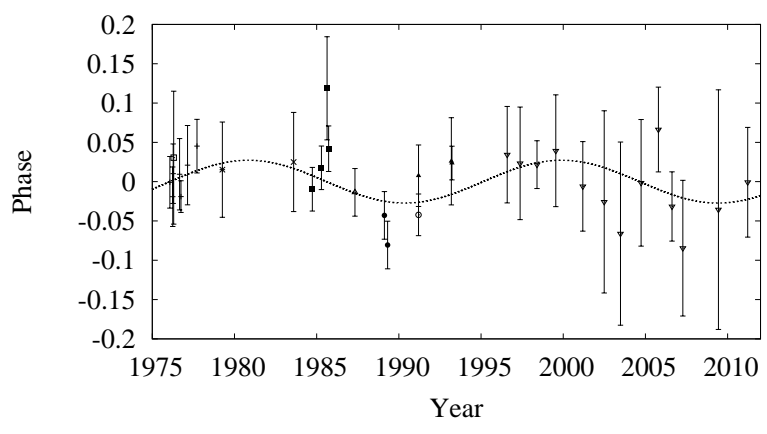

Fig. 5.- Differences (residuals) between the measured phase shifts and our quadratic solution. There seems to be a $19 \pm 2$ yr modulation $\left(\chi_{\text {red }}^{2}=\right.$ 0.70 ) which is roughly two times the $\approx 8.5 \pm 0.2 \mathrm{yr}$ sinusoidal curve as proposed by Tan et al. (1991). ephemeris to arrive at

$$
\Phi(t)=\Phi_{0}+\frac{\Delta P}{\left(P_{\text {fold }}\right)^{2}} t+\frac{1}{2} \frac{\dot{P}}{\left(P_{\text {fold }}\right)^{2}} t^{2} .
$$

Here $\Phi(t)$ is the measured shift over the time $t=T_{N}^{\max }-T_{\text {fold }}$, where $T_{N}^{\max }$ is the time of maximum of the $N$-th cycle and $T_{\text {fold }}=$ BJD $_{\text {TDB }} 2442803.63600$ corresponds to the offset in the ephemeris from Tan et al. (1991) and $P_{\text {fold }}=(685.0118 / 86400)$ days is the period of the same ephemeris. The phase offset is given by $\Phi_{0}=\left(T_{0}-T_{\text {fold }}\right) / P_{\text {fold }}$ and the period difference is $\Delta P=P_{0}-P_{\text {fold }}$. $T_{0}, P_{0}$ and $\dot{P}$ are the true offset, period and period derivative of the real ephemeris of $4 \mathrm{U} 1820-30$, which we want to determine. To finally calculate them we approximate Eq. 3 to the dataset as given in Table1 (see Fig. 4 and Fig. 5 for the residuals).

As shown in Fig. 5 the residuals seem to be compatible $\left(\chi_{\text {red }}^{2}=0.70\right)$ to a sinusoidal modulation having a $19 \pm 2 \mathrm{yr}$ period. This is approximately two times the $\approx 8.5 \pm 0.2 \mathrm{yr}$ sinusoidal period which was solely identified by Tan et al. (1991) and interpreted as an artifact subject to the sparse time 
coverage of the data. However, our dataset covers more than twice the time interval than theirs and it indicates an intrinsic origin of the source rather than an observational bias. We postpone the interpretation of Fig. 5 to future studies.

This yields a period of $P=$ $(685.01197 \pm 0.00003) \mathrm{s}$ and derivative of $\dot{P}=(-1.15 \pm 0.06) \times 10^{-12} \mathrm{~s} \mathrm{~s}^{-1} \quad$ (at $T_{0}=$ BJD $\left._{\mathrm{TDB}} 2442803.63608 \pm 0.000094\right)$. This translates into $\dot{P} / P=(-5.3 \pm 0.3) \times 10^{-8} \mathrm{yr}^{-1}$. The reduced $\chi^{2}$ value centers close to one, i.e $\chi_{\text {red }}^{2}=0.88$. The $95 \%$ confidence interval for the period derivative is $-1.279 \times 10^{-12} \mathrm{~s} \mathrm{~s}^{-1} \leq$ $\dot{P} \leq-1.019 \times 10^{-12} \mathrm{~s} \mathrm{~s}^{-1}, \quad$ respectively $-5.89 \times 10^{-8} \mathrm{yr}^{-1} \leq \dot{P} / P \leq-4.69 \times 10^{-8} \mathrm{yr}^{-1}$. Our $\dot{P} / P$ value is equal to the one found by van der Klis et al. (1993a) and is also consistent with the value from Chou \& Grindlay (2001). However, we lowered the error by at least a factor of four and can rule out any positive period derivative with a significance of $>17 \sigma$. The fact that the decrease of the period must be faster than Chou \& Grindlav (2001) claimed was already suspected by Zdziarski et al. (2007) and Levine et al. (2011).

In order to check whether the quadratic function approximates the data more accurately than the linear regression $\left(\chi_{\text {red }}^{2}=9.81\right)$, we performed an F-test which passed the $99.99 \%$ confidence level which we enforced. A cubic fit $\left(\chi_{\text {red }}^{2}=0.91\right)$ on the other hand failed the F-test at the same 99.99\% confidence level. Using our RXTE data alone is not sufficient to determine $\dot{P}$, since the F-test for the quadratic fit $\left(\chi_{\text {red }}^{2}=0.35\right)$ vs. the linear fit $\left(\chi_{\text {red }}^{2}=0.39\right)$ fails at the $99.99 \%$ confidence level. Still, the obtained value of $\dot{P} / P=$ $(-4.1 \pm 2.7) \times 10^{-8} \mathrm{yr}^{-1}$ is in agreement with the result from the combined dataset, although with an unacceptable large error value.

The updated ephemeris can now be written as:

$$
\begin{aligned}
T_{N}^{\max }=\mathrm{BJD}_{\mathrm{TDB}} 2442803.63608 \pm 0.000094 \\
\\
+\left(\frac{685.01197 \pm 0.00003 \mathrm{~s}}{86400 \mathrm{~s} / \mathrm{d}}\right) \times N+ \\
+\left((-4.6 \pm 0.3) \times 10^{-15}\right) \times N^{2}
\end{aligned}
$$

\section{Interpretation}

As already presented in the introduction, the theoretically expected value for the period derivative is positive and should, according to Rappaport et al. (1987), have a value of $\dot{P} / P>$ $8.8 \times 10^{-8} y r^{-1}$. Their arguments are based on Roche overflow from the secondary to the primary neutron star. Prodan \& Murrav (2012) discussed internal effects, which could also lower the intrinsic period derivative predicted by Rappaport et al. (1987). Although these effects would decrease the value, but they would not change its sign. Based on the fact that many other predictions made by Rappaport et al. (1987) are in good agreement with the measurements, it is reasonable to assume that the intrinsic period derivative is indeed positive (Prodan \& Murrav (2012)). It has to been investigated whether additional internal effects, like Applegate's Gravitational Quadrupole Coupling (GQC) (Applegate 1992) model, which successfully explains the negative period derivative of the pulsar PSR J2051-0827 (Lazaridis et al. 2011), can also explain the negtive period derivative.

However, our approach bases on external effects, which are also known to explain negative period derivatives. The most straightforward explanation for the negative period derivative is a Doppler shift caused by gravitational acceleration of 4U 1820-30 towards us. This idea was already discussed for 4U 1820-30 by van der Klis et al. (1993b), Chou \& Grindlav (2001) and Prodan \& Murrav (2012). The theoretical idea stems from Phinney (1992) who studied this effect as means to measure globular cluster potentials with the help of period derivatives from pulsars. Giersz \& Heggie (2011) applied this method convincingly in their Monte-Carlo modeling of the Milky Way globular cluster 47 Tucanae. We follow their approach here.

Phinney (1992) found the following relation for globular clusters:

$$
\frac{\dot{P}_{\text {int }}}{P} \leq \frac{\dot{P}_{\mathrm{obs}}}{P}+\frac{a_{c}}{c}+\frac{a_{G}}{c}+\frac{\mu^{2} D}{c}
$$

where $\dot{P}_{\text {int }}$ is the intrinsic period derivative in the rest frame, $\dot{P}_{\text {obs }}$ the observed period derivative, $a_{c}$ the acceleration due to the cluster potential, $a_{G}$ the acceleration due to the galaxy, $\mu$ the proper motion and $\mathrm{D}$ the distance to the cluster. The last term is the Shklovskii effect (Shklovskii 1970) which is positive but usually sub-dominant. The value for galactic acceleration for NGC 6624 is 
around $\frac{a_{G}}{c} \approx-5.7 \times 10^{-11} \mathrm{yr}^{-1}$ (applying Equation 2.3 from Phinney 1992) and can therefore be neglected.

Following Phinnev (1992), the expected period derivative change in a cluster potential is:

$$
\frac{a_{c}}{c}=-\frac{1}{c} \frac{G M(<r)}{r^{2}} \frac{l}{r},
$$

where $M(<r)$ is the cluster mass within the radius $r$ and $l$ is the distance along the line-of-sight (LOS) from the source to the line which is perpendicular to the LOS and which passes right through the center of the globular cluster. If the pulsar lies behind the center, $l$ is positive and hence the Doppler shift is negative.

The maximal possible acceleration at a given cluster radius depends on the mass distribution within the cluster. For a cored mass profile with a core radius $r_{c}$, Phinnev (1993) estimated the maximal acceleration to be

$$
\frac{a_{c, \max }}{c} \approx \frac{3 \sigma\left(R_{\perp}\right)^{2}}{2 c\left(r_{c}^{2}+R_{\perp}^{2}\right)^{1 / 2}}
$$

with $\sigma\left(R_{\perp}\right)$ being the line-of-sight velocity dispersion and $R_{\perp}$ being the projected distance. Using this method, Prodan \& Murray (2012) calculated the cluster acceleration to be $\frac{a_{\mathrm{c}, \max }}{c}=$ $1.3 \times 10^{-9} \mathrm{yr}^{-1}$ at the assumed position of $4 \mathrm{U}$ 1820-30. This value is significantly too low to be able to explain the observed period change. But their estimate of the acceleration assumes NGC 6624 to have a cored density profile. However, as already stated, NGC 6624 appears to be in deep core collapse and therefore shows a central cusp. Based on Fokker-Planck models of van der Klis et al. (1993b), Chou \& Grindlav (2001) estimated the gravitational acceleration on $4 \mathrm{U} 1820-30$ to be $\frac{a_{c, \max }}{c} \approx 7.9 \times 10^{-8} \mathrm{yr}^{-1}$. This value would be sufficient to explain the observed period derivative by assuming a negligible contribution from the internal positive period derivative. However, Chou \& Grindlav (2001) used the projected distance $0.02 \mathrm{pc}$ of $4 \mathrm{U} 1820-30$ to the center of NGC 6624 which is nowadays considered to be outdated by more recent observations. Using HST / ACS imaging data, Goldsbury et al. 2010) have redetermined the center of NGC 6624 to be slightly offset from previous HST determinations, which puts $4 \mathrm{U} 1820-30$ at a projected radius of
$0.046 \mathrm{pc}$ from the center. Since the modeling of the cluster acceleration on $4 \mathrm{U} 1820-30$ is very sensitive to this value, we here made a new modeling approach based on the latest observational data on NGC 6624.

\subsection{Modeling of NGC 6624}

Based on available observational constraints, we generated dynamical models of NGC 6624 to estimate the gravitational acceleration on $4 \mathrm{U}$ 1820-30 and on three further pulsars in NGC 6624, for which period derivatives have been measured (Lvnch et al. 2012). In Table2 we list the relevant properties of these three pulsars together with the corresponding data from $4 \mathrm{U} 1820-30$. What is striking with these four targets is that their period derivatives have the same magnitude, which could be interpreted of being affected by the same gravitational pul 2. Moreover, all four of them lie within about $0.5 \mathrm{pc}$ (projected) radius of the cluster center.

To create the models, we used the McLusTER code 3 Küpper et al. 2011). The observational constraints were given through the pulsar data, an extended surface brightness profile (Novola \& Gebhardt 2006), and radial velocity measurements of Prvor et al. (1989) and Valenti et al. (2011).

Novola \& Gebhardt (2006) combined HST/WFPC2 photometry of the center of NGC 6624 with ground-based wide-field data of Trager et al. (1995) to create a V-band surface brightness profile extending from 0.26 arcsec to 76 arcsec, which corresponds to $0.01-3.0 \mathrm{pc}$. We fit a cuspy Nuker profile (Lauer et al. 1995) to this surface brightness data by least squares minimization, where we used the uncertainties given by Novola \& Gebhardt (2006) as weights (thick black line in the upper row panels of Fig. (6). The Nuker surface density profile has the form

$I(R)=2^{(\eta-\gamma) / \alpha} I_{0}\left(\frac{R_{c}}{R}\right)^{\gamma}\left(1+\left(\frac{R}{R_{c}}\right)^{\alpha}\right)^{(\gamma-\eta) / \alpha}$

where $I_{0}$ is a scale surface density, $\gamma$ and $\eta$ are

\footnotetext{
${ }^{2}$ For an environment independent explanation for the pulsar shifts see Lvnch et al. (2012).

${ }^{3}$ https://github.com/ahwkuepper/mcluster.git
} 


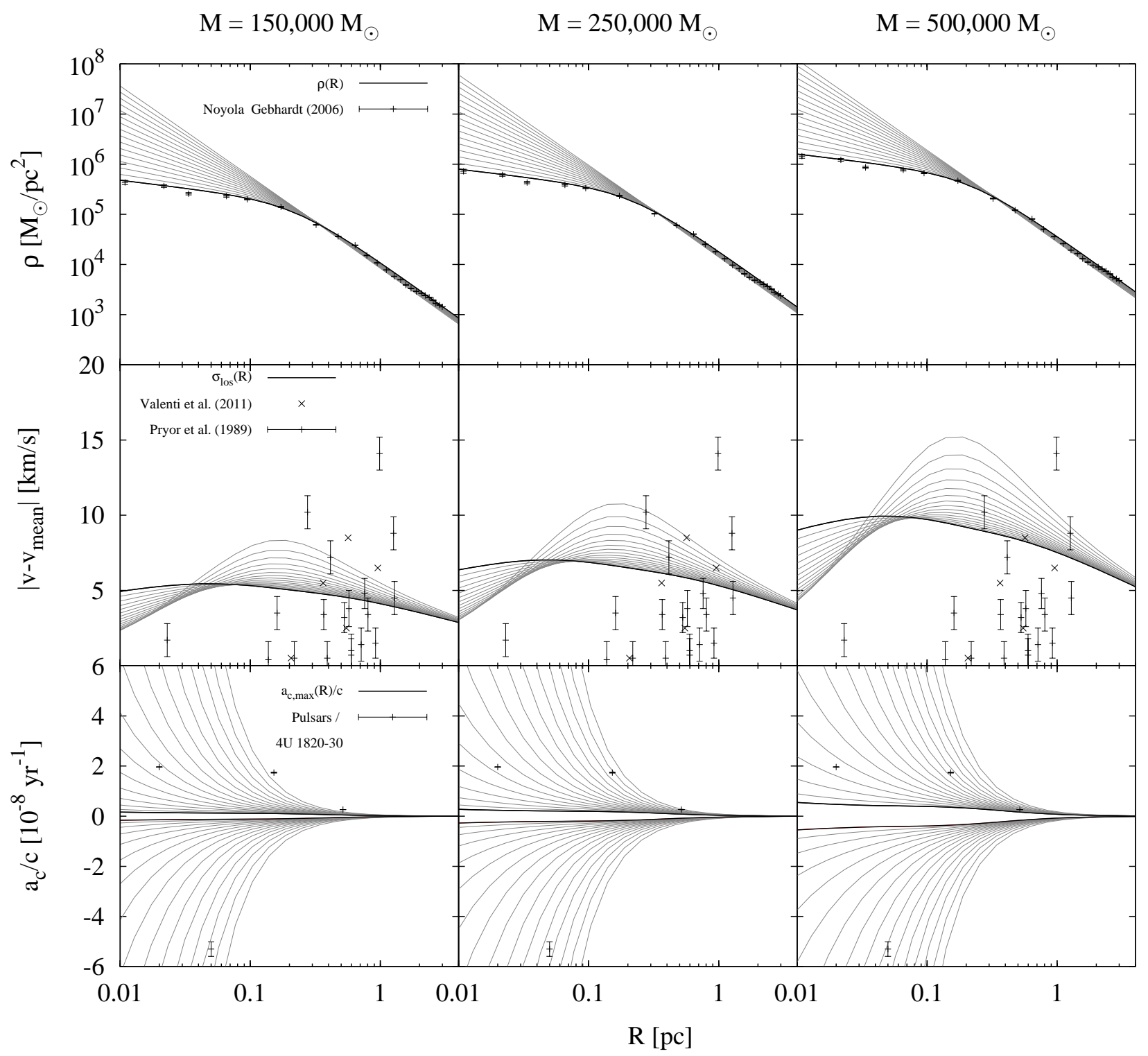

Fig. 6.- Comparison of cluster models of $1.5 \times 10^{5} \mathrm{M}_{\odot}$ (left column), $2.5 \times 10^{5} \mathrm{M}_{\odot}$ (middle column), and $5.0 \times 10^{5} \mathrm{M}_{\odot}($ right column), with observational data. Top row: The density profile of the models were generated using NGC 6624's observed surface brightness profile and assuming that mass follows light (thick black line) or that the mass density profile slope is steeper than the surface brightness slope of 0.32 towards the cluster center with slopes of 0.4 to 1.8 (gray lines). Middle row: Line-of-sight velocity dispersions for the models compared to 23 radial velocities of probable NGC 6624 member stars. Ideally, about one third of the measurements should lie above the respective dispersion profile. Bottom row: maximum acceleration within the cluster along the line-of-sight at a given radius for the cluster models compared to the period derivatives of the three pulsars and 4U 1820-30. Only very cuspy density distributions can cause the observed Doppler shift of 4U 1820-30. 


\begin{tabular}{cccc} 
Name & $\mathrm{R}[\operatorname{arcsec}]$ & $\mathrm{R}[\mathrm{pc}]$ & $\dot{P} / P\left[10^{-8} \mathrm{yr}^{-1}\right]$ \\
\hline \hline $\mathrm{J} 1823-3021 \mathrm{~A}$ & 0.52 & 0.020 & $1.9636 \pm 0.0006$ \\
$\mathrm{~J} 1823-3021 \mathrm{~B}$ & 13.46 & 0.516 & $0.263 \pm 0.003$ \\
$\mathrm{~J} 1823-3021 \mathrm{C}$ & 8.39 & 0.152 & $1.74 \pm 0.02$ \\
4U 1820-30 & 1.30 & 0.050 & $-5.3 \pm 0.3$ \\
\hline
\end{tabular}

Table 2: List of pulsars with known period derivatives in NGC 6624. The corresponding values for 4U 182030 were also added. List compiled from data from Lynch et al. (2012), Goldsbury et al. (2010), Zhang et al. (2011) and this work. See also accompanying Fig. 7 .

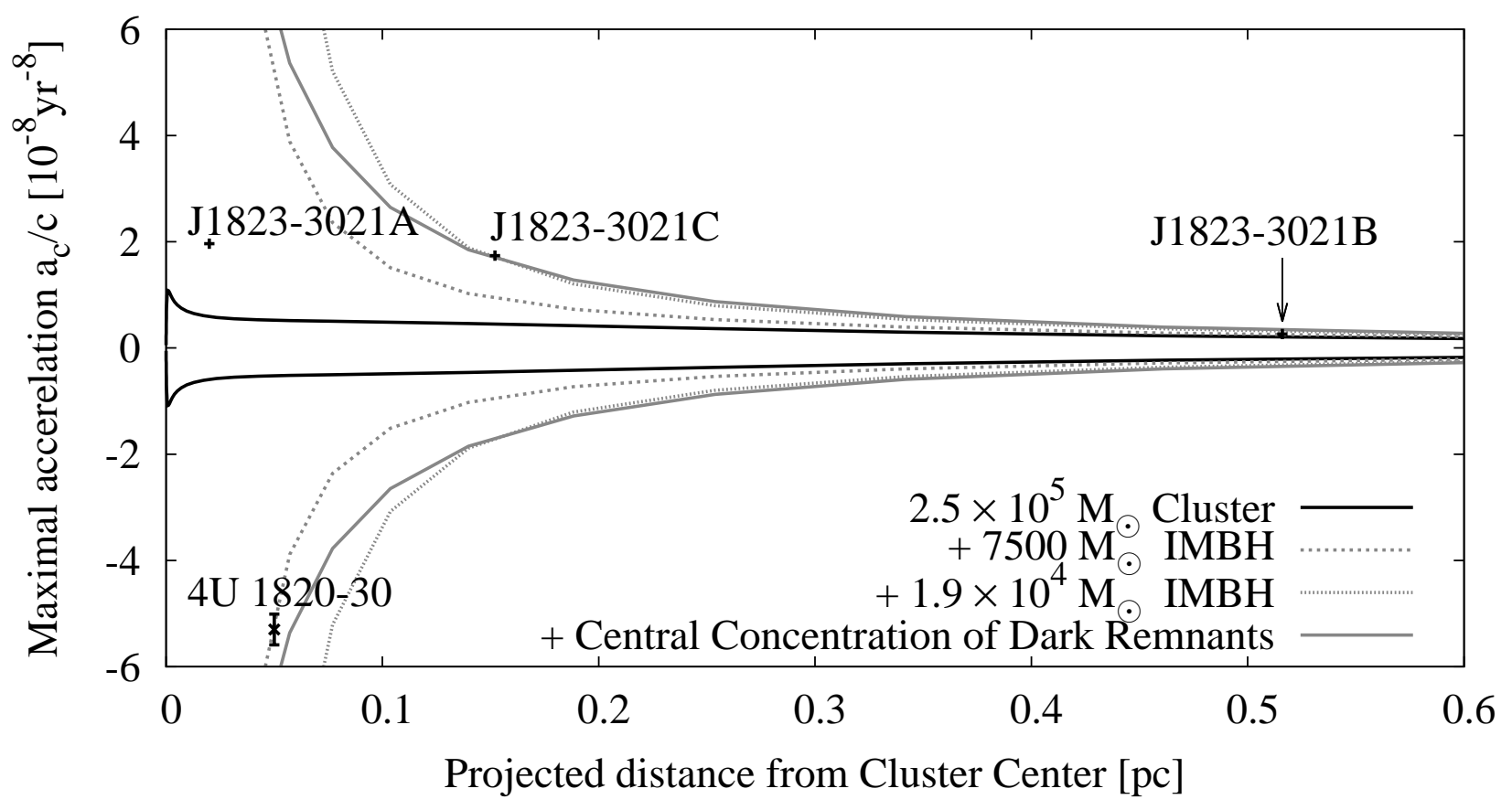

Fig. 7.- Expected maximal cluster acceleration depending on the projected radial position in the cluster, based on the cluster model with $2.5 \times 10^{5} \mathrm{M}_{\odot}$ in which the mass profile follows the observed surface brightness profile (thick black line). Also shown is this model but with an additional IMBH of $7500 \mathrm{M}_{\odot}$ (dashed line) or $1.9 \times 10^{4} \mathrm{M}_{\odot}($ dotted line), respectively. Alternatively, we show a cluster model with a central concentration of dark remnants (grey line), i.e. for which the mass profile deviates from the light profile. A central slope of the mass profile of 1.7 can explain the observed period derivatives. 
two power-law slopes, and $R_{c}$ is the break radius, where the profile transits from one slope into the other. We set the transition parameter $\alpha=2$. The resulting values were $\gamma=0.31$ for the inner profile slope, $\eta=1.85$ for the outer profile slope and a break radius of $R_{c}=0.22 \mathrm{pc}$, similar to the values found by Novola \& Gebhardt (2006), who used a King profile (King 1966). To create a mass profile from that, we first deprojected the profile into 3D assuming spherical symmetry, and then created a corresponding mass profile assuming that mass follows light in the cluster, i.e. that the cluster is not mass-segregated (in Section 4.4 we are going to drop this assumption to show that the cluster core may harbor a core of dark remnants). We then scaled this profile to a given total mass within a radius of $30 \mathrm{pc}$, that is between the tidal radius estimated by Lugger et al. (1987) of $26.8 \mathrm{pc}$ and the estimate from the modeling of Pryor et al. (1989) of about $33 \mathrm{pc}$. From this mass profile we got a velocity dispersion profile by solving the Jeans equation, assuming virial equilibrium and velocity isotropy. The latter seems to be appropriate, as Prvor et al. (1989) find that radially anisotropic dynamical models give poorer fits to the data.

From this $3 \mathrm{D}$ velocity dispersion profile we computed the line-of-sight (LOS) velocity dispersion profile following Binney \& Mamon (1982). We compared the resulting LOS velocity dispersion profile of these models against $18 \mathrm{DAO} / \mathrm{CFHT}$ radial velocities of probable NGC 6624 member stars from Pryor et al. (1989) and 5 radial velocities measured by Valenti et al. (2011) with high-resolution Keck/NIRSPEC data (middle row panels of Fig. 6). For this purpose we calculated projected distances from NGC 6624's center at $\mathrm{RA}=18: 23: 40.51$, Dec $=-30: 21: 39.7$ (J2000, Goldsburv et al. 2010) to the coordinates given in Valenti et al. (2011). Their data yields a velocity dispersion of $\approx 6 \mathrm{~km} / \mathrm{s}$ between $0.2 \mathrm{pc}$ and 1.0 pc projected distance from the center. For the Pryor et al. (1989) radial velocity data we used the radial distances from the cluster center as stated in their Table 2, due to a lack of proper coordinates. Together their data sets give a systemic velocity of $v_{\text {mean }}=53.5 \mathrm{~km} / \mathrm{s}$ and a velocity dispersion of $\sigma \approx 5.5 \mathrm{~km} / \mathrm{s}$ within $1.3 \mathrm{pc}$ projected distance. For a good fitting model, about $68 \%$ of the measured velocities should lie below the LOS velocity dispersion profile.
To scale the cluster light profile to a mass profile we need the mass-to-light ratio of the cluster population or its total mass. Unfortunately, the total mass of NGC 6624 is relatively unconstrained, as neither its total integrated magnitude nor its distance modulus are well measured. Hence, we can only collect and compare estimates based on various approaches: From multi-mass King model fitting, Pryor et al. (1989) find a mass between $100,000 \mathrm{M}_{\odot}$ and $220,000 \mathrm{M}_{\odot}$, and global massto-light ratios, $M / L_{V}$, between 1.9 and 2.3. The cluster's integrated absolute V-band magnitude is -7.49 mag (Harris 1996), which translates into a cluster luminosity of $L / L_{\odot}=10^{\left(M_{V, \odot}-M_{V}\right) / 2.5} \approx$ 85,000 . Thus, using a global mass-to-light ratio $M / L_{V}=1-3$ would yield a mass of $8.5 \times 10^{4} \mathrm{M}_{\odot}$ to $2.5 \times 10^{5} \mathrm{M}_{\odot}$. However, NGC 6624 is embedded in a rich foreground and background of bulge and disk stars. In addition, the uncertainty in NGC 6624's distance has a strong influence on this estimate. If we just take the central surface brightness profile of Novola \& Gebhardt (2006), which is less affected by contamination, transform it into a luminosity density profile and scale it to the luminosity profile of our test models, we find that a model with $1.5 \times 10^{5} \mathrm{M}_{\odot}$ matches the data well if we assume a global mass-to-light ratio of about 3 . However, this estimate involves an extrapolation of the Noyola data from 3 to $30 \mathrm{pc}$, and also involves the uncertain cluster distance which significantly affects this mass estimate. As a conclusion from these exercises we take that the mass of NGC 6624 should be somewhere between $1 \times 10^{5} \mathrm{M}_{\odot}$ and $2.5 \times 10^{5} \mathrm{M}_{\odot}$.

A mass of $M=250,000 \mathrm{M}_{\odot}$ yields an estimated Jacobi radius, $r_{J}$, of $25-30$ pc for NGC 6624 . For this estimate we assume a circular Galactic orbit and a local circular velocity of between $170-220 \mathrm{~km} / \mathrm{s}$ at the assumed Galactocentric radius, $R_{G C}$, of $1.2 \mathrm{kpc}$, and we use

$$
r_{J}=\left(\frac{G M}{2 \Omega^{2}}\right)^{1 / 3}
$$

where $\Omega=V_{c} / R_{G C}$ is the local angular velocity (e.g. Küpper et al. 2010). For this mass we get a line-of-sight velocity dispersion of $\sigma_{\mathrm{LOS}} \approx 6 \mathrm{~km} / \mathrm{s}$ within a radius of $1.3 \mathrm{pc}$ (central panel of Fig. 6). $M=250,000 \mathrm{M}_{\odot}$ therefore seems to be a reasonable assumption for the mass of NGC 6624 .

However, the maximal acceleration at the po- 
sition of $4 \mathrm{U} 1820-30$ of this model is $\frac{a_{\mathrm{c}, \max }}{c} \approx$ $2.3 \times 10^{-9} \mathrm{yr}^{-1}$. This value is an order of magnitude too low, but also the maximal accelerations for the three other pulsars are too low, as can be seen from the thick black line in the middle panel of the lower row of Fig. 7 In this figure we have plotted the distance-dependent expected maximal acceleration from our dynamical model together with the position of the four targets. To test if a higher cluster mass could solve the problem, we scaled the Novola \& Gebhardt (2006) to the cluster's mass profile using an unrealistic global massto-light ratio of 10 (top panel in the left column of Fig. 6). In this case we get a cluster mass of $5 \times 10^{5} \mathrm{M}_{\odot}$ within a radius of $30 \mathrm{pc}$. But even for this high mass-to-light ratio (which might be biased through the uncertain distance modulus), the maximal acceleration at the position of $4 \mathrm{U} 1820-30$ would only be $\frac{a_{\mathrm{c}, \max }}{c} \approx 4.6 \times 10^{-9} \mathrm{yr}^{-1}$ (lower left panel of Fig. 6). The same applies to the maximal accelerations at the radii of the two inner pulsars. Higher cluster masses would make the observed velocity dispersion rather unlikely (see the thick black line in the middle left panel of Fig. 6). Apparently, the cluster profile as given through the surface brightness profile can neither explain the acceleration of $4 \mathrm{U} 1820-30$ nor of the other three pulsars. This suggests that there is either an additional, significant source of gravity close to these objects or that the mass-to-light ratio of NGC 6624 is significantly higher in the center than in the outer parts. In the following we discuss these possible scenarios: A stellar mass dark remnant, an IMBH or a central concentration of dark remnants.

\subsection{Stellar mass dark remnant}

The negative observed time period might be explained by the radial gravitational acceleration towards a nearby, so far undetected, dark remnant. This dark object, passing close to $4 \mathrm{U}$ 1820-30, can be a white dwarf, a neutron star or a stellar mass black hole. In order to constrain the allowed parameter range, we performed computations with the $N$-body software CATENA (Pflamm-Altenburg \& Kroupa 2006). In this way we investigated the dynamical stability of the $4 \mathrm{U}$ 1820-30 system which we used as the main criterion. 4U 1820-30 was set up as documented by Prodan \& Murray (2012), with a third main se- quence star in a narrow orbit around the inner WD-NS binary. The additional companion scenario is the only theory which can explain the 171 day period so far.

We then tested the dynamical stability of this triple in the gravitational field of an external perturber, i.e. a forth object. The mass range of this dark remnant was varied between $1-100 \mathrm{M}_{\odot}$. Depending on the actual mass of the perturber, the required distance $d_{\text {eff }}$ to $4 \mathrm{U} 1820-30$ (i.e. center of mass) was calculated with the help of Eq. 6] by assuming a radial line of sight acceleration. Fig. 8 illustrates the dependency of a non-zero line-ofsight inclination.

The orientation of the velocity vector of the dark remnant with respect to the triple was randomly generated. The only boundary condition was that without gravitational interaction the dark remnant would pass $4 \mathrm{U} 1820-30$ with a minimal distance of $d_{\text {eff }}$. The velocity of the remnant was drawn from a normal distribution around the $3 \mathrm{D}$ velocity dispersion of the cluster $(6 \mathrm{~km} / \mathrm{s})$. The initial distance of the perturber was more than 10 times the distance $d_{\text {eff. }}$. In order to increase the statistical significance, this procedure was repeated ten times for every considered perturber mass.4.

The results are shown in Fig. 9, were we plotted the fraction of cases where the triple remains intact i.e. the survival rate against the dark remnant mass. It is apparent that for masses greater than $6 \mathrm{M}_{\odot}$, the LMXB would not survive such a flyby, leaving us with relatively light remnants. Analogous simulations with a dark remnant in a bound orbit yield comparable results.

Light remnants must be close to the $4 \mathrm{U} 1820$ 30 system in order to generate the required acceleration. However, the time they can maintain the required acceleration is strongly reduced. The timespan $T_{a}$, where the acceleration is in the $5 \sigma$-region of our measured value, varied between $\sim 20 \mathrm{yr}$ (for a $1 \mathrm{M}_{\odot}$ dark remnant) to $\sim 160 \mathrm{yr}$ (for a $5.5 \mathrm{M}_{\odot}$ dark remnant), which is illustrated in Fig. 10, These numbers give a rather quantitative than qualitative result, but they indicate that the chance to see a flyby right now is considerably low.

\footnotetext{
${ }^{4}$ The whole computational timescale was of the order of 1.5 month on a conventional quad-core CPU.
} 


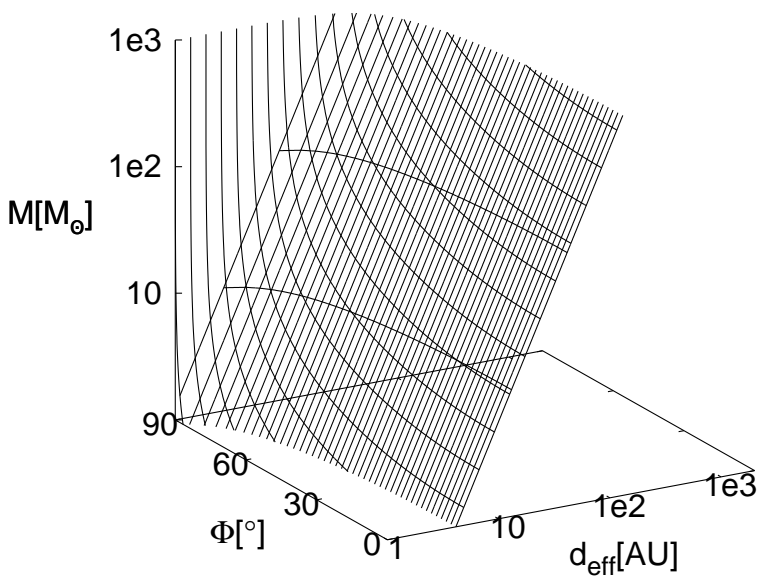

Fig. 8. - For a given line-of-sight inclination $\Phi$ and dark remnant mass $M$, the plot illustrates the required distance $d_{\text {eff }}$ to achieve the gravitational acceleration.

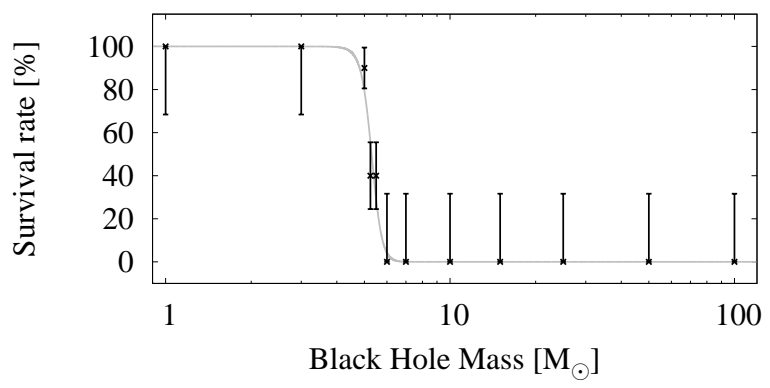

Fig. 9.- Survival rate of 4U 1820-30 after a flyby of a dark remnant of different masses. The solid line shows an exponential fit of the form $f(x)=1 /(1+\exp (a \cdot x+b))$ through the data. Error bars for mass ranges $1-3,6-100 \mathrm{M}_{\odot}$ are Poisson errors, while for the mass range $3-6 \mathrm{M}_{\odot}$ the errors were determined using bootstrapping.

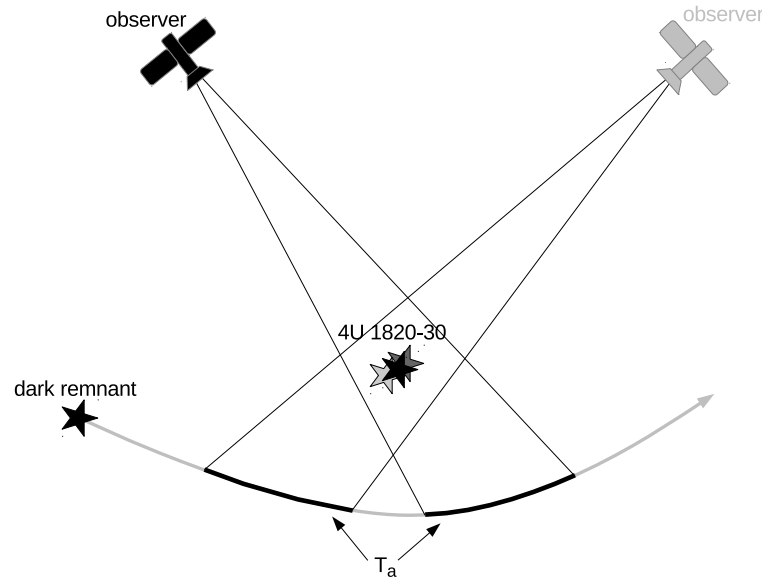

Fig. 10.- Illustration of a typical flyby scenario. $T_{a}$ is the time where the acceleration is in the $5 \sigma$ region of our measured value. Note that there exist two periods where independent observers could measure the effect.

Hence, the flyby scenario is a rather short lived phenomenon, which can not be excluded yet, but by measuring the second derivative, $\ddot{P}$, of the period, one could in fact constrain it. However, the chance that the other three pulsars are also experiencing a flyby event is highly unlikely.

\subsection{IMBH}

Lützgendorf et al. (2013) present evidence for the existence of intermediate mass black holes (IMBHs) in galactic globular clusters. At first glance such an object would be a considerable solution; the negative period derivative of $4 \mathrm{U} 1820$ 30 could be explained by the radial gravitational acceleration (Eq. 6) towards an IMBH of at least $7500 \mathrm{M}_{\odot}$ (see Fig. 7]).

The idea of NGC 6624 having an IMBH in its center is not a new one: already Bahcall (1976) suspected that NGC 6624 could be harboring a massive black hole.

However, there are certain arguments which contradict this idea: First of all, the period shifts of the three pulsars can only be explained by the $1 / r$-potential of a very massive IMBH (Fig. 7) in excess of $19000 \mathrm{M}_{\odot}$.

Furthermore, assuming the triple nature for $4 \mathrm{U}$ 1820-30 as proposed by Prodan \& Murrav (2012), the most stringent argument against an $\mathrm{IMBH}$ 
causing the negative period derivative is related to its stability. In the previous subsection, we analyzed the stability of the $4 \mathrm{U} 1820-30$ system. We found that massive objects in excess of $6 \mathrm{M}_{\odot}$ would disrupt the triple (as can be seen in Fig. 9). However, the IMBH would have a mass at least 3000 times greater than that limit. This would imply that the period derivative we observe for $4 \mathrm{U} 1820$ 30 is a unique event with a short duration as the triple would be destroyed by tidal forces rather quickly.

\subsection{A Central Concentration of Dark Remnants}

As an alternative to the above scenarios, we may consider that the assumption of a constant mass-to-light ratio across the cluster, which we used for generating our dynamical models, is not fulfilled. That is, the surface brightness profile may not be a good tracer of the mass distribution. The central mass-to-light ratio may be significantly enhanced by dark remnants, such as white dwarfs, neutron stars and/or stellar mass black holes, which sank into the center via mass segregation. Spitzer (1969) showed that heavier objects like black holes can form a dynamically decoupled subsystem within a star cluster, if their total mass is a significant fraction of the total cluster mass. In star clusters that contain a high fraction of massive dark remnants, the result of such a Spitzer instability is a high central concentration of dark mass, which inflates the central mass-tolight ratio (see Banerjee \& Kroupa 2011 for the extreme cases of dark star clusters). Using idealized two-component systems, Breen \& Heggie (2013) showed that the lifetime of such a dynamical subsystem is longer the smaller the difference in the masses of the heavier objects and the lighter objects are. That is, a subsystem of many lower-mass dark remnants, such as neutron stars or white dwarfs, would survive for a longer time than a subsystem of black holes with masses of several solar masses, and would therefore be more likely to have survived until the present day within an old globular cluster like NGC 6624 .

We may assume that such a dark remnant concentration has formed inside the $0.22 \mathrm{pc}$ break radius of the radial surface brightness profile, and that therefore inside this radius the surface brightness profile is flatter than the mass density profile.
To see the effect of such a dark remnant cusp inside NGC 6624, we generated models with deviating power-law slopes inside the break radius, which is equivalent to having a radially increasing mass-tolight ratio towards the center. The surface brightness profile shows an inner slope of 0.31 , we varied this slope in steps of 0.1 up to the outer profile slope of 1.85 . The resulting mass density profiles, line-of-sight velocity dispersion profiles and maximum acceleration profiles for all three model masses are shown in Fig. 6 .

When we include a high amount of dark mass in the center of our model of NGC 6624 with a mass of $250,000 \mathrm{M}_{\odot}$, and assume that the mass density profile within the break radius has a slope of about 1.7 instead of the 0.31 observed for the surface brightness profile, all three period shifts of the inner pulsars can be explained by the same phenomenon, invoking less of a coincidence than the other scenarios. The amount of (dark) mass necessary would be of the order of $70,000 \mathrm{M}_{\odot}$ and would be limited to within the break radius of 0.22 pc. Interestingly, even our model with a mass of $150,000 \mathrm{M}_{\odot}$ could nearly reproduce the pulsar Doppler shifts if the mass density profile followed the same power-law slope of $\approx 1.8$ inside the break radius like outside the break radius. This would correspond to a dark remnant mass of about $55,000 \mathrm{M}_{\odot}$ inside the break radius.

Both configurations would imply that about $27 \%-37 \%$ of the cluster mass is locked up in dark remnants inside the cluster's break radius, yielding a dark remnant density of about $10^{6} \mathrm{M}_{\odot} / \mathrm{pc}^{3}$. This significant contribution from dark remnants has to be in accordance with stellar evolution models. To check this we used MCLuster for generating aged stellar populations. If we assume that NGC 6624 was born with a regular initial mass function (e.g. Kroupa 2001), and neglect dynamical evolution for a crude estimate, the dark remnant mass fraction at present day, i.e. after 12 Gyr of stellar evolution, would be $30 \%$. This is in good agreement with our findings. Most of this mass would come from white dwarfs (21\%), and $9 \%$ of the total mass would be in neutron stars and black holes. However, dynamical evolution (cluster mass loss, preferential loss of lowmass stars, and ejections from the core as a consequence of few-body interactions), as well as birth kicks for neutron stars and black holes, will alter 
the contribution from dark remnants to the total cluster mass over time. Based on its present-day mass function, Marks et al. (2012) suggested that NGC 6624 may have been born with a top-heavy IMF, which would certainly help to produce such a dark-remnant enriched cluster. Performing detailed $N$-body computations of NGC 6624 to show how a star cluster can form such a dark-remnant feature would be desirable, but is beyond the scope of this paper.

The dark mass concentration would have consequences for the radial velocity dispersion of stars within the central cusp of NGC 6624: The cuspy density profiles show an enhanced peak LOS velocity dispersion inside the break radius (gray lines in the middle row panels in Fig. 60). Interestingly, Zaggia et al. (1992) find with integrated spectroscopy of the inner $\approx 0.16 \mathrm{pc}$ that the velocity dispersion of NGC 6624 in this part is of the order of $9 \mathrm{~km} / \mathrm{s}$. In contrast to that, most radial velocity measurements for NGC 6624 were outside this radius where the effect of the central density cusp is weaker. For a central slope of 1.7 and a cluster mass of $250,000 \mathrm{M}_{\odot} 4$ out of 23 stars would still lie above the LOS velocity dispersion profile. For a slope of 1.8 and a mass of $150,000 \mathrm{M}_{\odot} 7$ out of 23 , or $30 \%$, could lie outside the predicted profile. The peak dispersion of this model is $\approx 8.5 \mathrm{~km} / \mathrm{s}$. We therefore conclude that this model reflects the current data reasonably well.

Interestingly, a similar configuration of a dark remnant concentration has been discussed by Novola et al. (2008) for the center of the Milky Way globular cluster $\omega$ Centauri. They estimated that $10^{4}$ dark remnants would be necessary within a radius of $0.05 \mathrm{pc}$ of $\omega$ Centauri's core to reproduce the observations. In the end, they favored an $\mathrm{IMBH}$ as the explanation for the observed surface density and velocity dispersion profile of this cluster. They argued that such a high concentration of dark remnants could not have been able to form in $\omega$ Centauri as its present-day half-mass relaxation time is so long. However, the authors did not take into account that a Spitzer instability significantly speeds up the formation of such a dark remnant core. For 47 Tuc, which has a nearly identical metallicity and age as NGC 6624 (Heasley et al. 2000), Giersz \& Heggie (2011) find that dark remnants make up about $34 \%$ of 47 Tuc's total mass and that the central dark remnant fraction is even larger than 50\%. Gebhardt \& Fischer (1995) came to similar conclusions fitting Jeans models to the much better and more abundant observational data for 47 Tuc.

The idea that NGC 6624 could harbor a high amount of dark remnants has also been addressed by Grabhorn et al. (1992), who used FokkerPlanck models to analyze the dynamical state of NGC 6624. Following an independent approach from ours, their resulting model had a total mass of $M_{t o t}=1.5 \times 10^{5} \mathrm{M}_{\odot}$, in which about $37 \%$ of the total mass, or $M_{\text {dark }}=5.6 \times 10^{4} \mathrm{M}_{\odot}$, was bound in dark remnants. If NGC 6624 had been able to keep a fair amount of its white dwarfs, black holes and neutron stars in the past, we may in fact be observing the result of a Spitzer instability in the center of NGC 6624. The further investigation of this cluster is therefore of fundamental interest and may even yield valuable insights into the nature of dark remnant formation.

\section{Conclusion}

By reducing 16 years of RXTE data (Section 2), we solved one important question concerning the, in many aspects extreme, low-mass X-ray binary 4U 1820-30: Its period derivative is indeed negative with a value of $\dot{P} / P=(-5.3 \pm 0.3) \times$ $10^{-8} \mathrm{yr}^{-1}$. However, by doing so, we created a new puzzling question, as this is completely contrary to the expectations for a neutron star accreting mass from a Roche lobe-filling white dwarf in an isolated binary system.

Fortunately, the $4 \mathrm{U} 1820-30$ system is not in isolation but in the very center of the Milky Way globular cluster NGC 6624. We therefore present some possible interpretations of this observation in Section 4. We argue that this period shift is primarily due to gravitational acceleration from a non-luminous object. This could either be an intermediate-mass black hole (IMBH) in the center of NGC 6624, a nearby dark stellar remnant on a flyby, or a central dark remnant concentration within NGC 6624.

We showed that an IMBH and a heavy dark remnant would disrupt the complex 4U 182030 system, so that its negative period derivative would purely be a chance observation. The same could be the case for a lighter dark remnant, since 
the time scales are quite short. However, if three more pulsars within NGC 6624 are considered for the interpretation of the $4 \mathrm{U} 1820-30$ observations, it appears more likely that all four of them are being strongly accelerated within the gravitational field of NGC 6624. In order to explain all four period derivatives, an extended concentration of non-luminous mass within the center of NGC 6624 would be necessary. We therefore suggest that a dark subsystem of black holes, neutron stars and/or white dwarfs may have formed via Spitzer instability in NGC 6624. Thus, a more detailed study of NGC 6624's dynamical state could yield valuable insights into the formation mechanisms of dark remnants.

A better understanding of the internal processes of LMXBs may explain the negative period derivative $4 \mathrm{U}$ 1820-30 without the need for a Doppler shift from NGC 6624 (e.g. Postnov \& Shakura 1987, Podsiadlowski et al. 2002). However, as we have shown here, the presence of additional matter in the cluster core can explain not just $4 \mathrm{U}$ 1820-30's period derivative, but also the strong Doppler shift observed for the pulsars at larger radii.

To shed more light on the problem, it would be helpful if other X-ray satellite missions could continue to observe 4U 1820-30. The RXTE satellite ceased science operations on 3 January 2012. As presented in Section 2 the data we used had quite a coarse temporal resolution and most of the observations were not designed to observe the $685 \mathrm{sec}$ periodicity. We hope that further observations will not only reduce the error on the period derivative but also help to evaluate the second period derivative. This value would allow to probe if the negative period derivative is a local transient phenomenon or if, as the pulsar period derivatives seem to indicate, is due to concentration of some sort of non-luminous mass in NGC 6624's center. Also, the determination of the period derivative of so far unconstrained pulsars (J1823-3021D,E,F) would give further insights to the structure of NGC 6624. Especially, a measurement of the period derivative of pulsar J1823-3021D would be important, as it lies at the same (projected) distance from the center of NGC 6624 as J1823-3021C does. Another possibility to improve our knowledge of the dark component of NGC 6624 could be the search and analysis of additional short-period LMXBs like the recently discovered COM_Star1 (Dalessandro et al. 2014).

We thank Snezana Prodan for valubale discussion during her visit of the SPODYR research group in Bonn. We would also like to thank Jay Strader and the referee for helpful comments. MB would like to acknowledge support through DFG grant KR 1635/39-1. AHWK would like to acknowledge support through DFG Research Fellowship KU 3109/1-1 and from NASA through Hubble Fellowship grant HST-HF-51323.01-A awarded by the Space Telescope Science Institute, which is operated by the Association of Universities for Research in Astronomy, Inc., for NASA, under contract NAS 5-26555.

Results provided by the ASM/RXTE teams at MIT and at the RXTE SOF and GOF at NASA's GSFC.

\section{REFERENCES}

Anderson, S. F., Margon, B., Deutsch, E. W., Downes, R. A., \& Allen, R. G. 1997, ApJ, 482, L69

Applegate, J. H. 1992, ApJ, 385, 621

Arons, J., \& King, I. R. 1993, ApJ, 413, L121

Bahcall, N. A. 1976, ApJ, 204, L83

Banerjee, S., \& Kroupa, P. 2011, ApJ, 741, L12

Binney, J., \& Mamon, G. A. 1982, MNRAS, 200, 361

Breen, P. G., \& Heggie, D. C. 2013, MNRAS, 432, 2779

Canizares, C. R., Grindlay, J. E., Hiltner, W. A., Liller, W., \& McClintock, J. E. 1978, ApJ, 224, 39

Chou, Y., \& Grindlay, J. E. 2001, ApJ, 563, 934

Dalessandro, E., Pallanca, C., Ferraro, F. R., et al. 2014, ApJ, 784, L29

Eastman, J., Siverd, R., \& Gaudi, B. S. 2010, PASP, 122, 935

Gebhardt, K., \& Fischer, P. 1995, AJ, 109, 209

Giersz, M., \& Heggie, D. C. 2011, MNRAS, 410, 2698 
Goldsbury, R., Richer, H. B., Anderson, J., et al. 2010, AJ, 140, 1830

Grabhorn, R. P., Cohn, H. N., Lugger, P. M., \& Murphy, B. W. 1992, ApJ, 392, 86

Grindlay, J., Gursky, H., Schnopper, H., et al. 1976, ApJ, 205, L127

Grindlay, J. E. 1988, in IAU Symposium, Vol. 126, The Harlow-Shapley Symposium on Globular Cluster Systems in Galaxies, ed. J. E. Grindlay \& A. G. D. Philip, 347-363

Harris, W. E. 1996, AJ, 112, 1487

Heasley, J. N., Janes, K. A., Zinn, R., et al. 2000, AJ, 120,879

Jahoda, K., Markwardt, C. B., Radeva, Y., et al. 2006, ApJS, 163, 401

Jahoda, K., Swank, J. H., Giles, A. B., et al. 1996, in Society of Photo-Optical Instrumentation Engineers (SPIE) Conference Series, Vol. 2808, Society of Photo-Optical Instrumentation Engineers (SPIE) Conference Series, ed. O. H. Siegmund \& M. A. Gummin, 59-70

King, I. R. 1966, AJ, 71, 276

Kroupa, P. 2001, MNRAS, 322, 231

Küpper, A. H. W., Kroupa, P., Baumgardt, H., \& Heggie, D. C. 2010, MNRAS, 401, 105

Küpper, A. H. W., Maschberger, T., Kroupa, P., \& Baumgardt, H. 2011, MNRAS, 417, 2300

Lauer, T. R., Ajhar, E. A., Byun, Y.-I., et al. 1995, AJ, 110,2622

Lazaridis, K., Verbiest, J. P. W., Tauris, T. M., et al. 2011, MNRAS, 414, 3134

Levine, A. M., Bradt, H., Cui, W., et al. 1996, ApJ, 469, L33

Levine, A. M., Bradt, H. V., Chakrabarty, D., Corbet, R. H. D., \& Harris, R. J. 2011, ApJS, 196,6

Lugger, P. M., Cohn, H., Grindlay, J. E., Bailyn, C. D., \& Hertz, P. 1987, ApJ, 320, 482

Lützgendorf, N., Kissler-Patig, M., Gebhardt, K., et al. 2013, A\&A, 552, A49
Lynch, R. S., Freire, P. C. C., Ransom, S. M., \& Jacoby, B. A. 2012, ApJ, 745, 109

Marks, M., Kroupa, P., Dabringhausen, J., \& Pawlowski, M. S. 2012, MNRAS, 422, 2246

Morgan, E. H., Remillard, R. A., \& Garcia, M. R. 1988, ApJ, 324, 851

Noyola, E., \& Gebhardt, K. 2006, AJ, 132, 447

Noyola, E., Gebhardt, K., \& Bergmann, M. 2008, ApJ, 676, 1008

Pflamm-Altenburg, J., \& Kroupa, P. 2006, MNRAS, 373, 295

Phinney, E. S. 1992, Royal Society of London Philosophical Transactions Series A, 341, 39

Phinney, E. S. 1993, in Astronomical Society of the Pacific Conference Series, Vol. 50, Structure and Dynamics of Globular Clusters, ed. S. G. Djorgovski \& G. Meylan, 141

Piotto, G., King, I. R., Djorgovski, S. G., et al. 2002, A\&A, 391, 945

Podsiadlowski, P., Rappaport, S., \& Pfahl, E. D. 2002, ApJ, 565, 1107

Postnov, K. A., \& Shakura, N. I. 1987, Soviet Astronomy Letters, 13, 122

Priedhorsky, W., \& Terrell, J. 1984, ApJ, 284, L17

Prodan, S., \& Murray, N. 2012, ApJ, 747, 4

Pryor, C., McClure, R. D., Fletcher, J. M., \& Hesser, J. E. 1989, AJ, 98, 596

Rappaport, S., Ma, C. P., Joss, P. C., \& Nelson, L. A. 1987, ApJ, 322, 842

Sansom, A. E., Watson, M. G., Makishima, K., \& Dotani, T. 1989, PASJ, 41, 591

Shaposhnikov, N., Jahoda, K., Markwardt, C., Swank, J., \& Strohmayer, T. 2012, ApJ, 757, 159

Shklovskii, I. S. 1970, Soviet Ast., 13, 562

Smale, A. P., Mason, K. O., \& Mukai, K. 1987, MNRAS, 225, 7P

Smale, A. P., Zhang, W., \& White, N. E. 1997, ApJ, 483, L119 
Sosin, C., \& King, I. R. 1995, AJ, 109, 639

Spitzer, Jr., L. 1969, ApJ, 158, L139

Stella, L., Priedhorsky, W., \& White, N. E. 1987a, ApJ, 312, L17

Stella, L., White, N. E., \& Priedhorsky, W. 1986, in Bulletin of the American Astronomical Society, Vol. 18, Bulletin of the American Astronomical Society, 1048

Stella, L., White, N. E., \& Priedhorsky, W. 1987b, ApJ, 315, L49

Strohmayer, T. E., \& Brown, E. F. 2002, ApJ, 566,1045

Tan, J., Morgan, E., Lewin, W. H. G., et al. 1991, ApJ, 374, 291

Trager, S. C., King, I. R., \& Djorgovski, S. 1995, AJ, 109, 218

Valenti, E., Origlia, L., \& Rich, R. M. 2011, MNRAS, 414, 2690

van der Klis, M., Hasinger, G., Verbunt, F., et al. 1993a, A\&A, 279, L21

van der Klis, M., Hasinger, G., Dotani, T., et al. 1993b, MNRAS, 260, 686

Zaggia, S. R., Capaccioli, M., Piotto, G., \& Stiavelli, M. 1992, A\&A, 258, 302

Zdziarski, A. A., Gierliński, M., Wen, L., \& Kostrzewa, Z. 2007, MNRAS, 377, 1017

Zhang, Z., Gilfanov, M., Voss, R., et al. 2011, A\&A, 533, A33

This 2-column preprint was prepared with the AAS LATEX macros v5.2. 